\title{
Image analysis e wine tourism destination: la prospettiva dell'enoturista
}

\author{
PAOLA SCORRANO ${ }^{* \star}$ MONICA FAIT \\ PIERFELICE ROSATO $^{\star * \star *}$ LEA IAIA $^{* \star \star *}$
}

\begin{abstract}
Obiettivo del paper: L'obiettivo principale della ricerca consiste nell'individuare gli attributi che qualificano l'immagine delle destinazioni enoturistiche ed analizzarne il ruolo nella percezione dei wine tourist.

Metodologia: Facendo propri i principi della letteratura manageriale in materia di Destination Management e, nello specifico, di Destination Image, si è inteso adottare a livello metodologico il modello di misurazione proposto da Echtner e Ritchie (1991).

Risultati: Si è pervenuti ad una mappatura delle risorse che contribuiscono a qualificare l'immagine di una destinazione enoturistica, verificandone la rilevanza assegnata dagli enoturisti, con uno specifico focus sulle principali destinazioni enoturistiche internazionali.

Implicazioni pratiche: La mappatura generale alla quale si è pervenuti può costituire, se opportunamente contestualizzata, uno strumento per valutare l'ampiezza del percorso che un'area deve intraprendere per valorizzare i prerequisiti di base fino a renderli identificabili come un sistema enoturistico dai wine tourist.

Originalità e limiti della ricerca: Il principale elemento di originalità attiene alla scelta di applicare una metodologia di analisi consolidata in tema di immagine della destinazione ad un ambito, quello enoturistico, di crescente rilevanza in chiave di sviluppo turistico, ma ancora poco studiato ed approfondito, pur con il limite derivante dalla difficoltà di definire il campione secondo idonee regole statistiche che permettano una generalizzazione dei risultati.
\end{abstract}

Parole chiave: destination management; enoturismo; immagine

* Benchè il presente lavoro derivi da una comune opera degli autori, il paragrafo 1 è di Pierfelice Rosato, i paragrafi 2 e 4.2 di Paola Scorrano, i paragrafi 3 e 4.1 di Lea Iaia, il paragrafo 4.3 di Monica Fait ed il paragrafo 5 di tutti gli autori.

** $\quad$ Ricercatore di Economia e Gestione delle Imprese - Università del Salento e-mail: paola.scorrano@unisalento.it

**** Ricercatore di Economia e Gestione delle Imprese - Università del Salento e-mail: monica.fait@unisalento.it

***** Ricercatore di Economia e Gestione delle Imprese - Università del Salento e-mail: pierfelice.rosato@unisalento.it

****** Dottorando di Ricerca di Economia Aziendale - Università del Salento e-mail: lea.iaia@unisalento.it 
Purpose of the paper: The main objective of the research is to identify the drivers that define the image of wine tourism destinations, and analyze its role in the perception of the wine tourists.

Methodology: Embracing the principles of management literature in the field of Destination Management and, specifically, of Destination Image, the methodological measurement framework proposed by Echtner and Ritchie (1991) has been adopted.

Findings: A resource map was decided on, the aim of which was to qualify the image of a wine tourism destination. It checked the relevance given by wine tourists, with a specific focus on the main international wine tourism destinations.

Pratical implications: If properly contextualized, the general mapping represents a useful tool which is able to assess the magnitude of the path that an area should take to enhance basic prerequisites so as to make them identifiable as a system of wine tourism for the wine tourist.

Originality and research limits: The main element of originality in this research lies in the fact that it applies consolidated Destination Image analysis methodology to a wine tourism destination; indeed the importance of wine tourism has been increasing in terms of tourism development, nevertheless it has not been investigated in any great depth. However, there are inevitably limits arising from the difficulty in defining the samples in accordance with appropriate statistical rules that allow a generalization of the results.

Key words: destination management; wine tourism; image

\section{Ambito teorico della ricerca}

Negli anni si è assistito ad un crescente interesse di studiosi e policy makers per il tema del Tourism Destination Image in relazione alla rilevanza che tale aspetto riveste nella definizione dei processi di destination management. La natura image driven del settore turistico ha favorito la produzione, sin dalla metà degli anni Settanta, di contributi tesi ad evidenziare lo stretto legame esistente tra l'immagine dei luoghi ed il comportamento di scelta dei turisti (Hunt,1975). Nella letteratura manageriale internazionale il tema è stato affrontato assumendo quattro differenti prospettive (Elliot et al., 2011): la rilevanza che l'immagine di una destinazione assume nel processo di scelta del turista (Woodside e Lysonski, 1989; Pearce, 1982); la misurazione dell'immagine della destinazione attraverso la definizione di specifici modelli (Echtner e Ritchie, 1991); l'analisi del processo di formazione dell'immagine (Baloglu e McCleary, 1999); l'individuazione degli effetti positivi per la destinazione in termini di attrattività e competitività associati all'immagine positiva di cui gode (Tapachai e Waryszak, 2000).

Il presente contributo s'inserisce nel filone di studi attinente la misurazione dell'immagine delle destinazioni turistiche. Nello specifico si è inteso adattare, come avremo modo di esplicitare meglio nel prosieguo del lavoro, il modello proposto da Echtner e Ritchie alla misurazione dell'immagine delle principali destinazioni enoturistiche internazionali al fine di evidenziarne il processo di emersione ed i relativi drivers (Echtner e Ritchie, 1991). 
La scelta di analizzare le destinazioni enoturistiche trova giustificazione nell'esistenza di una consolidata nicchia turistica globale riconducibile al wine tourism (Hall et al., 2000), fenomeno che assume una rilevanza crescente a livello mondiale in termini di flussi turistici associati e di capacità di determinare specifici modelli di comportamento ed esigenze espresse (Mitchell et al., 2000). In tale prospettiva d'indagine, la destinazione enoturistica viene intesa come un'area geografica che, sulla base di definite e codificate risorse specifiche riconducibili al tourist terroir (Hall et al. 2000; Peters, 1997; Hall e Mitchell, 2002; Telfer, 2001), sia nelle condizioni di avviare processi di destination management atti ad individuare segmenti di mercato che riconoscano la bontà di un'offerta di destinazione wine - related appositamente concettualizzata (Rosato e Scorrano, 2010).

Su tali presupposti, le destinazioni turistiche ed enoturistiche sono chiamate a riflettere sul tema della propria immagine puntando non soltanto sugli aspetti funzionali e simbolici, ma soprattutto su quei tratti, prevalentemente di natura immateriale, che le rendono uniche (Echtner e Ritchie, 1993; Morrison e Anderson, 2002; Rosato e Iazzi, 2008) e le differenziano chiaramente rispetto ad altre destinazioni concorrenti (Cai, 2002).

Sin dalle sue prime teorizzazioni (Gunn, 1972; Mayo, 1973), il tema dell'immagine ha assunto una significativa rilevanza nell'ambito della letteratura manageriale sulle destinazioni (Blain et al., 2005; Kaplanidou e Vogt, 2003; Nandan, 2005; Lassar et al., 1995), diventando un fattore critico di successo capace di agire in termini di promozione, distribuzione e sviluppo dei prodotti turistici (Sonmez e Sirakaya, 2002; Pikkemaat, 2004), oltre che di apportare benefici in termini di distintività, redditività e capacità di sviluppare innovazione (Pencarelli e Gregori, 2009).

Se, infatti, i turisti considerano la destinazione al pari di un qualsiasi altro prodotto, valutandone gli attributi tangibili ed intangibili (Clifton, 2003; Murphy, 1998; Ward et al., 1999; Beerli e Martín, 2004; Florek, 2005) attraverso un processo organico in cui convergono elementi funzionali, simbolici ed esperienziali (Chon 1990; Foster e Jones, 2000; Gartner, 1993; Kim e Yoon, 2003; Walmsley e Young, 1998), è evidente che lo sviluppo di un'immagine positiva e altamente distintiva sia cruciale non solo in termini di identificazione e posizionamento della specifica destinazione turistica (Kapferer, 1997; Pappu et al., 2005; Ries e Trout, 1972), ma anche per la costruzione di un solido vantaggio competitivo (Baloglu e McCleary, 1999).

In questo senso, l'immagine di una destinazione agisce sui suoi potenziali visitatori in quanto:

- rappresenta una semplificazione del gran numero di informazioni connesse con una destinazione (Kotler et al., 1993), incidendo positivamente sull'economia cognitiva del consumatore;

- guida il comportamento individuale (Barich e Kotler, 1991) in quanto assume un ruolo cruciale: 
a) nel processo decisionale di scelta e di acquisto (Goodrich, 1978; Hunt, 1975; Pike e Ryan, 2004; Tapachai e Waryszak, 2000; Cai et al., 2004; Dana e McClearly, 1995; Leisen, 2001; Tasci e Kozak, 2006; Jago et al., 2003; Morgan et al., 2002; Ooi, 2004);

b) in fase di formulazione del giudizio globale di soddisfazione o insoddisfazione dell'esperienza vissuta (Chon, 1990);

c) in merito alle intenzioni di riacquisto e sul passaparola (Bignè et al., 2001);

- offre garanzie qualitative e fornisce la promessa di una memorabile esperienza di viaggio, legandola specificamente ad una destinazione. Ciò, da un lato, permette al turista di acquisire un quadro di attese che riduce la percezione del rischio, dall'altro offre al management la base per la costruzione di una salda unique selling proposition (Blain et al., 2005);

- riflette in maniera organica i valori, i prodotti, i servizi e le competenze dei diversi attori economici di una specifica area geografica (Govers et al., 2007), comunicandoli in maniera unitaria all'esterno (Buhalis, 2000) e incentivando lo scambio di risorse e competenze all'interno (Haugland et al., 2011);

- consolida il ricordo dell'esperienza e favorisce il richiamo alla memoria dei suoi aspetti più piacevoli (Ritchie e Ritchie, 1998).

Se il contribuito che l'immagine può fornire allo sviluppo e alla competitività di una destinazione è ampiamente condiviso, l'adozione di un approccio interdisciplinare di studio (Baloglu e McCleary, 1999; Hosany et al., 2006; Mackay e Fesenmaier, 2000; Uysal et al., 2000) ha reso la sua qualificazione teorica particolarmente complessa, impedendo di arrivare ad una definizione univoca (Gallarza et al., 2002).

Alcuni studi considerano l'immagine come un costrutto inconsapevole e sovraordinato rispetto ai singoli attributi che contribuiscono a definire l'offerta (Ahmed, 1991; Dadgostar e Isalo, 1995). Altri, invece, la inquadrano come un costrutto multi-attributo, analizzandone le tre componenti fondamentali, gerarchicamente interrelate, che intervengono per la sua formazione: la componente cognitiva, quella conativa e quella affettiva (Gartner, 1993; Baloglu e McCleary, 1999). In tali modelli, le valutazioni cognitive si riferiscono alle credenze e alle conoscenze relative agli attributi di una specifica destinazione, quelle conative fanno riferimento alla volontà di visitarla, mentre quelle affettive fanno riferimento al complesso di sentimenti ed emozioni associato alla destinazione e fungono da variabile tra la valutazione cognitiva e la costruzione dell'immagine globale che si forma nel momento in cui si valuta una determinata destinazione.

L'immagine, inoltre, è dinamica, perché soggetta a cambiamenti nel corso del tempo (Gartner e Hunt, 1987; Dann 1996) e relativistica, perché è un insieme di percezioni che variano anche a seconda dello spazio e della distanza geografica in cui esse si vengono a formare (Crompton, 1979; Min Han, 1989).

La numerosità delle definizioni presenti in letteratura e la multidimensionalità (Ryan, 2003) del concetto di "immagine di destinazione" rendono complessa anche la scelta del modello tramite cui misurarla (Dobni e Zinkhan, 1990; Martínez e de Chernatony, 2004), sebbene sia ampiamente riconosciuto come la determinazione 
dell'efficacia del brand costituisca un aspetto cruciale per garantire nel lungo periodo il successo delle politiche di promo-commercializzazione di una destinazione turistica (Blain et al., 2005).

A ciò si aggiunge la considerazione che, a causa dell'aumento della pressione competitiva, non è più sufficiente per una destinazione turistica costruire la propria immagine sulla qualità delle proprie strutture ricettive, la bellezza del proprio paesaggio e/o l'accoglienza delle persone: tali aspetti, infatti, sono venuti a configurarsi come dei point of parity (Baker, 2007), necessari ma non sufficienti per agire quali specifici attrattori nella scelta di un potenziale visitatore.

\section{Obiettivi della ricerca}

Sulla base dei presupposti teorici richiamati, il presente lavoro intende individuare gli attributi che qualificano l'immagine delle destinazioni enoturistiche ed analizzarne il ruolo nella percezione dei wine tourist.

La letteratura di marketing tende a definire l'immagine della destinazione con riferimento alla brand identity, cioè quello che la destinazione comunica di sé, ed alla brand image, ciò che il consumatore percepisce di essa (Kapferer, 1997). La prima è chiamata a fornire una direzione strategica, uno scopo e un significato a tutti gli elementi del brand, conferendo loro omogeneità ed incentivando le associazioni con la marca (Aaker, 1996), mentre la seconda indica quel complesso di costrutti e rappresentazioni mentali (Reynolds, 1985; Embacher e Buttle, 1989), pregiudizi favorevoli o non favorevoli (Parenteau, 1995), sentimenti ed impressioni, di natura cognitiva ed emotiva, associate nella memoria del consumatore ad uno specifico brand (Dobni e Zinkhan, 1990; Baloglu e McCleary, 1999).

L'ottica di osservazione qui adottata è quella della brand image (Keller, 1993; Kapferer, 1997) su cui si fonda la capacità dei consumatori di percepire le differenze tra destinazioni.

I sub-obiettivi che si intendono raggiungere sono:

1) pervenire ad una mappatura degli attributi che contribuiscono a qualificare l'immagine di una destinazione enoturistica, verificandone la rilevanza assegnata dagli enoturisti;

2) individuare le principali destinazioni enoturistiche internazionali e definirne l'immagine nella percezione degli enoturisti.

A tal fine si è inteso adattare il modello di Echtner e Ritchie (1991), il cui tratto fondamentale risiede nella constatazione che una destinazione deve essere favorevolmente differenziata $\mathrm{e} / \mathrm{o}$ positivamente posizionata nella mente del consumatore e che, nel valutare l'immagine di una destinazione, vadano considerate tre dimensioni fondamentali:

- Attributes Vs Holistic: è un percorso che ha inizio con l'individuazione dei singoli attributi direttamente connessi con la destinazione e termina con l'individuazione degli elementi che descrivono, in un'ottica sistemica, l'immagine mentale degli stessi; 
- Functional Vs Psychological characteristics: in tale dimensione una caratteristica concreta ed osservabile potrebbe subire un processo di astrazione psicologica e dunque essere valutata in relazione alla sensazione che suscita nel turista;

- Common Vs Uniqueness: consente di identificare gli elementi distintivi di una destinazione enoturistica, distinguendo fra quelli considerati comuni-generici a tutte le destinazioni e quelli unici della stessa.

\section{Metodologia}

Al fine di raggiungere gli obiettivi predetti è stato redatto un questionario validato tramite test pilota ed opportunamente revisionato - composto da10 domande aperte e a scelta multipla, articolato in tre sezioni.

La prima (domande 1-6) ha consentito di profilare i wine tourist che hanno partecipato all'indagine, rilevando i dati concernenti la nazionalità, l'età, il sesso, la professione. È stato loro chiesto, inoltre, di collocarsi nell'ambito di una specifica categoria di enoturisti, scegliendo tra le seguenti (Charters e Ali-Knight, 2002):

- wine expert, se in possesso di un'approfondita conoscenza sul settore vinicolo e di un'elevata esperienza in ambito enoturistico;

- wine enthusiast, se dispongono di una buona conoscenza sul vino, ma non possiedono una considerevole esperienza in ambito enoturistico;

- wine interested, se soltanto interessati al mondo del vino e dell'enoturismo ed aspirano, pertanto, a diventare enoturisti.

La seconda parte del questionario (domanda 7) ha inteso rilevare la notorietà delle destinazioni, chiedendo all'intervistato di specificare quali siano, a suo parere, le prime tre destinazioni enoturistiche mondiali.

L'obiettivo della terza e ultima sezione del questionario (domande 8-10) è stato quello di pervenire ad una mappatura degli attributi che qualificano l'immagine delle wine tourism destination. E stato, dunque, chiesto all'enoturista di fornire, con riferimento alle destinazioni in precedenza scelte, tre indicazioni (graduandole per importanza assegnata) circa le seguenti dimensioni:

a. images or characteristics: elementi che vengono in mente al turista nel momento in cui pensa alla destinazione;

b. atmosphere or mood: umore o stato d'animo che una data destinazione suscita nel turista. Esso può derivare da fattori che, sebbene concreti, portano con sé degli elementi di tipo simbolico-emozionale ed esperenziale;

c. distinctive or unique tourist attractions: elementi distintivi e/o unici che connotano la destinazione e la identificano in modo univoco.

La diffusione del questionario è avvenuta impiegando gli strumenti digitali del Web 2.0 con l'obiettivo precipuo di coinvolgere, su scala mondiale, il maggior numero di enoturisti e di wine-blogger. A tal fine, il link del questionario è stato più volte postato sulle pagine Facebook e Twitter di: 
- destinazioni notoriamente votate all'enoturismo, individuate sulla scorta di precedenti ricerche inerenti le destinazioni enoturistiche di eccellenza a livello mondiale (Scorrano, 2011a, 2011b), ad es. "Champagne Ardenne", "Napa Valley", "Sonoma Valley", "Tuscany", "La Rioja", etc.;

- soggetti ritenuti "aggregatori", per via della tematica trattata, ovvero turismo e/o vino, ad es. "International Wine Tourism Conference", "Châteauneuf du Pape", "Chianti Classico", "Destination Marlborough", "Chile Travel", "Slow Food International", "Touring Club Italiano", "Wine news", "Wine tourism news", etc.;

- topic in linea con il tema vino e/o turismo, ad es. "Vino", "Enoturismo", "Tourism", etc.;

- blogger nazionali ed internazionali.

Le attività di somministrazione dei questionari, osservazione dei tassi di risposta e conseguente sollecito per posta elettronica, post su Facebook e Twitter e ripetuto invio del questionario, sono state condotte nel periodo 15 maggio-15 giugno 2012; l'espletamento delle stesse ha consentito, mediante un campionamento di convenienza, di pervenire ad un numero di questionari compilati e ritenuti validi pari a 145 .

\section{Risultati della ricerca}

Di seguito viene proposta una sintesi dei dati primari raccolti, rielaborati ai fini dell'indagine in oggetto.

\subsection{II profilo dell'enoturista}

La rielaborazione della prima sezione del questionario ha permesso di pervenire alla seguente profilazione degli enoturisti.

Il campione dei wine tourist che ha partecipato all'indagine (cfr. figura 1) è composto equamente da uomini e donne (50\%), di età compresa fra i 18 ed i 50 anni $(80,6 \%)$, con un livello di istruzione prevalentemente universitario $(82,6 \%)$ e un impiego professionale $(24,2 \%)$. Dalla distinzione degli enoturisti sulla base della loro provenienza geografica si evince la prevalenza di viaggiatori di nazionalità italiana $(46,8 \%)$, seguiti da inglesi $(17,7 \%)$, californiani $(4,8 \%)$, polacchi $(4,8 \%)$ e spagnoli $(4,8 \%)$.

Dal punto di vista della conoscenza enoica e dell'esperienza enoturistica posseduta, i wine tourist sono equamente distribuiti fra coloro che posseggono un livello di preparazione ed un'esperienza enoturistica medio-alta (50\%) e coloro che, essendo fortemente interessati al tema, hanno intenzione di diventare dei wine tourist esperti. 
Fig. 1: Quadro sinottico campione enoturisti intervistati
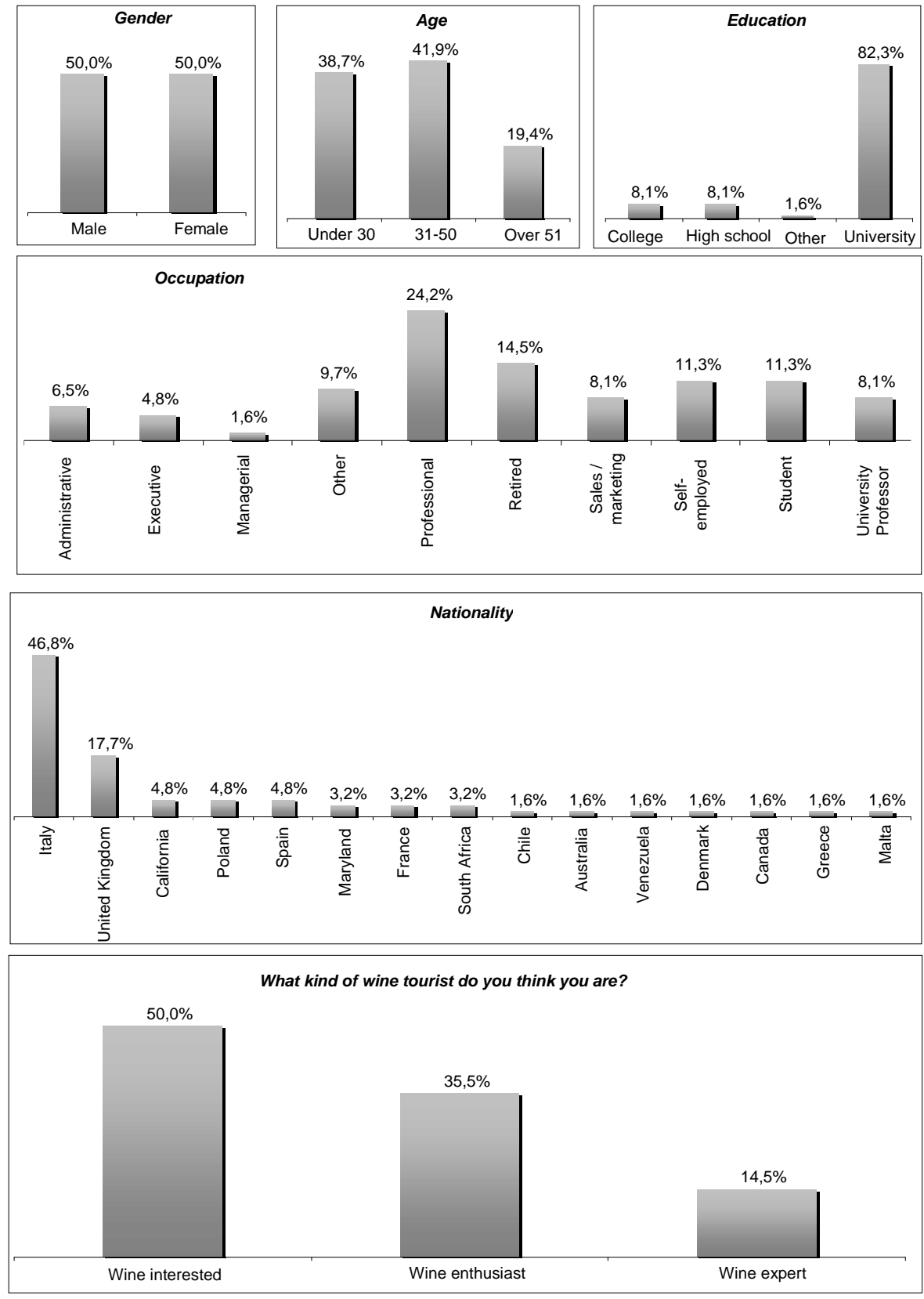

Fonte: ns. elaborazione 


\subsection{La rilevazione degli attributi dell'immagine di una generica wine tourism destination}

La rielaborazione delle risposte alle domande 8-9-10 ha permesso di pervenire alla mappatura degli attributi riferibili all'immagine di una generica destinazione enoturistica.

Con riferimento a ciascuna delle tre domande, si è proceduto con:

- estrapolazione dell'elenco dei termini utilizzati dagli intervistati, loro normalizzazione e sintesi semantica ${ }^{1}$;

- assegnazione, mediante congruo metodo a punteggio, di un valore a ciascuno dei predetti termini tenendo conto della frequenza e della rilevanza assegnata dall'enoturista;

- costruzione della matrice per la mappatura degli attributi dell'immagine (cfr. figura 2), adattando il modello di Echtner e Ritchie (1991).

Fig. 2: Destination image di una generica destinazione enoturistica

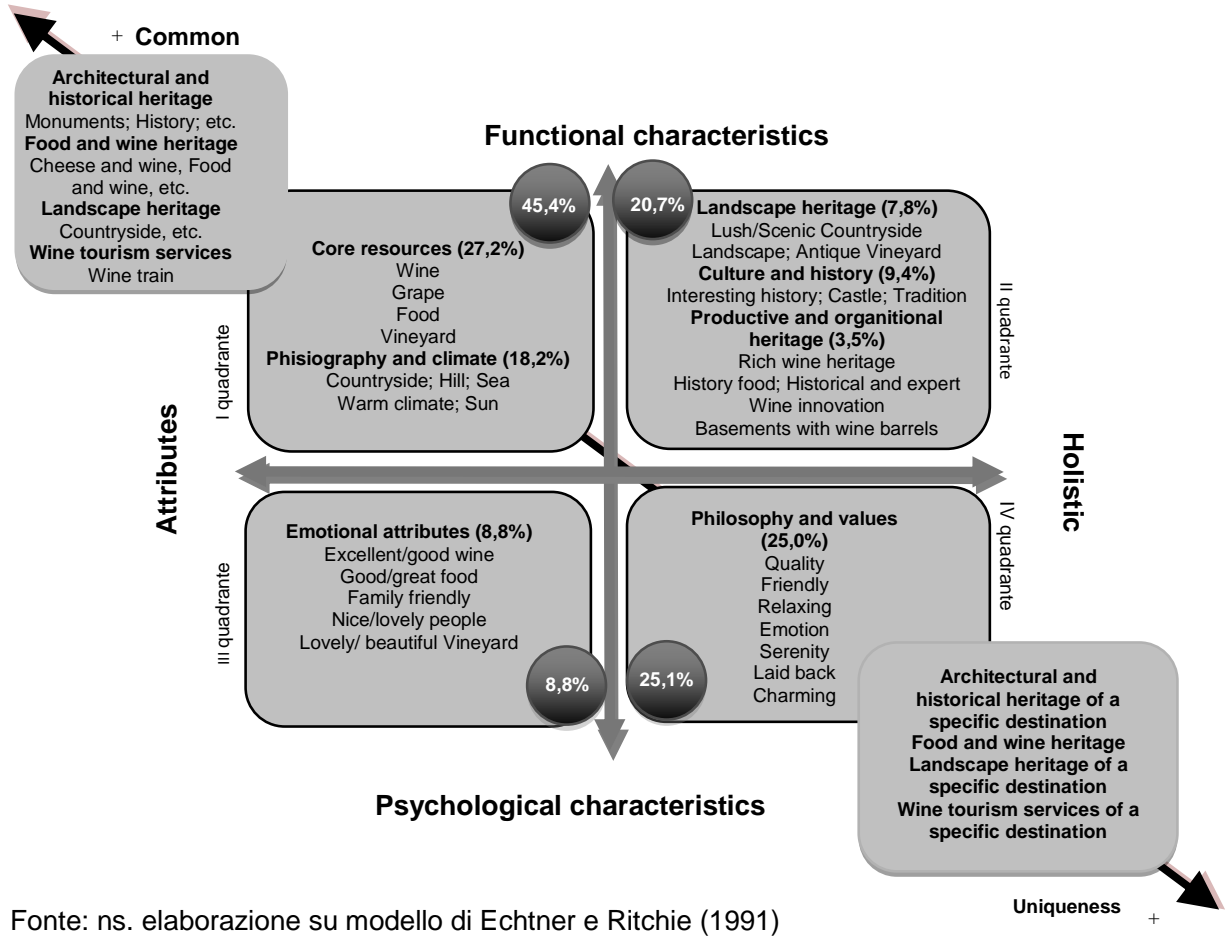

1 Conversione dei plurali in singolari, dei femminili in maschili, eliminazione di articoli, avverbi, pronomi, ecc. e raggruppamento delle parole considerando i sinonimi. 
Per la realizzazione della predetta matrice, in un primo momento si è tenuto conto delle dimensioni Images or characteristics e Atmosphere or mood poiché per entrambe è possibile operare lungo le direttrici Attributes Vs Holistic e Functional Vs Psychological characteristics. I termini con il punteggio maggiore sono stati analizzati in funzione della loro capacità di denotare una destinazione, in quanto componenti di base nel novero di quelle definibili enoturistiche. Gli stessi assumono valenza funzionale quando percepiti nello stesso modo da chiunque, senza l'attivazione di un percorso di astrazione psicologica. Tuttavia, qualora espressi in associazione con aggettivi o altri sostantivi, essi divengono indicativi di una rilevanza percettivo-cognitiva (psicologica) assegnata loro dall'enoturista. In tal modo si è giunti all'individuazione del primo e del terzo quadrante della matrice:

I quadrante - Functional Characteristics/Attributes: include i singoli elementi non associati ad aggettivi o altri nomi:

- wine, vineyard, grape, food, poiché intesi quali prerequisiti delle destinazioni enoturistiche ed altresì elementi comuni ad esse;

- countryside, hill, sun, sea, warm climate, in quanto elementi connessi al fattore territorio, immediatamente percepibili da chiunque, data la loro valenza funzionale.

III quadrante - Attributes/Psychological Characteristics: considera quei fattori che, seppur concreti, subiscono un processo di astrazione psicologica nella mente dell'enoturista, espressa attraverso l'associazione con aggettivi (ad esempio excellent/good + wine; good/great + food; beautiful/lovely + vineyard; nice/lovely + people; family + friendly).

In altre parole, ciò che rileva per il posizionamento in questo quadrante non è l'elemento in sé - wine, vineyard, ecc. - ma è la percezione - good, great, lovely, ecc.- che di esso ha l'enoturista.

In un secondo momento, la costruzione della matrice ha riguardato gli elementi che il wine tourist considera in chiave olistica, ovvero, quei fattori che connotano la destinazione enoturistica e ne aumentano l'attrattività, in quanto difficilmente replicabili ed imitabili da destinazioni concorrenti. Anche tali elementi possono avere caratteristiche funzionali, quindi immediatamente individuabili, così come essere oggetto di astrazione psicologica. Da tale valutazione derivano i quadranti II e IV della matrice. Più precisamente:

II quadrante - Functional Characteristics/Holistic: riguarda quegli elementi concreti che vengono percepiti dagli enoturisti in un'ottica olistica, poiché valutati all'interno di un sistema più ampio, caratterizzato da legami storici, artistici, architettonici, paesaggistici e di tradizione produttiva. Pertanto, sono stati inseriti termini come: "wine" quando utilizzato all'interno di frasi del tipo "rich wine heritage" o "basements with wine barrels"; "history food", "tradition", "antique vineyard", "interesting history", "historical and expert" poiché rilevano l'importanza assegnata alle tradizioni ed alla storia di un territorio vinicolo; "lush/scenic/beautiful" associati a "countryside" e "landscape", in quanto evidenziano la valenza scenica del paesaggio di campagna e dell'architettura agreste. 
IV quadrante - Holistic/Psychological Characteristics: rappresenta la sintesi degli attributi sensoriali che vengono evocati nell'immaginario e nello stato d'animo del wine tourist; i termini maggiormente impiegati, infatti, sono: "friendly", "relaxing", "emotion", "serenity", "laid back", "charming" e "quality".

In linea generale si rileva, dunque, che il processo di formazione dell'immagine di una destinazione enoturistica passa attraverso un percorso che prende avvio da elementi comuni e generici, i quali divengono fattori in grado di creare un differenziale competitivo allorquando:

1. la loro dimensione funzionale viene percepita in modo sistemico,

2. gli stessi vengono sottoposti ad un processo di astrazione psicologica singola,

3. l'astrazione psicologica che ne viene fatta avviene all'interno di una dimensione olistica.

Il successivo step della rielaborazione si è incentrato sull'esame della dimensione Distinctive or unique tourist attractions, con lo scopo di individuare gli elementi che generano nell'immagine del consumatore una perfetta identità con la destinazione cui riferiscono. La peculiarità di tale analisi è da rinvenire nella collocazione che gli stessi elementi hanno nella matrice, ovvero esclusivamente sulla direttrice Common Vs Uniqueness. I dati rivelano che, nel processo di creazione dell'immagine della destinazione, gli enoturisti associano al concetto di attrazione distintiva ed unica, con immediatezza e senza alcun input esterno da parte dell'intervistatore, fattori legati al contesto storico-geografico di un'area (patrimonio architettonico, città d'arte, patrimonio storico, ecc.) così come elementi comuni (wine, countryside e food).

Con riferimento a ciascuno dei quattro quadranti della predetta matrice si è inoltre ritenuto di esplicitare, in modo sintetico, la rilevanza assegnata dagli enoturisti agli attributi dell'immagine individuati.

Pertanto, la dimensione Functional Characteristics/Attributes ha conseguito un peso complessivo del 45,4\%: in particolare il 27,2\% è attribuibile alle Core resources ed il $18,2 \%$ alle Phisiography and climate. Questo ribadisce che nella mente del consumatore la destinazione enoturistica viene identificata attraverso l'associazione tra elementi naturali e prerequisiti comuni alle wine region. Essi, in seguito all'avvio di un processo di astrazione psicologica, divengono Emotional attributes, conseguendo una rilevanza dell' $8,8 \%$; quando invece sono oggetto di una valutazione olistica (quadrante Functional Characteristics/Holistic) raggiungono un peso complessivo del $20,7 \%$, attribuibile nello specifico a: Landscape heritage (7,8\%), Culture and history $(9,4 \%)$ e Productive and organizational heritage $(3,5 \%)$.

Di particolare interesse è la rilevanza, pari al 25\%, assegnata agli elementi Philosophy and values (quadrante Holistic/Psychological Characteristics), la quale evidenzia l'importanza degli elementi immateriali connessi all'atmosfera ed allo stato d'animo che una destinazione suscita nell'enoturista.

Le considerazioni sopra richiamate, seppur non generalizzabili, sono indicative di quanto la valorizzazione sistemica di elementi apparentemente comuni possa creare differenziazione nell'immaginario del wine tourist. 


\subsection{La rilevazione degli attributi dell'immagine delle principali wine destination}

L'analisi della domanda 7 ha consentito di:

a) individuare le principali destinazioni enoturistiche internazionali,

b) definirne l'immagine nella percezione degli enoturisti.

La metodologia adottata è la seguente:

a) tenendo conto del numero di volte che in totale una destinazione è stata menzionata e del numero di volte in cui la stessa è stata nominata per $1^{\circ}-2^{\circ}-3^{\circ}$, si è proceduto ad assegnare un valore pari a: 3 se la destinazione è stata menzionata per prima, 2 se indicata al secondo posto, 1 se indicata come terza. La graduatoria della notorietà delle destinazioni così ottenuta pone in evidenza che quelle più note sono anche le destinazioni di più antica tradizione vinicola France, Bordeaux, Italy e Tuscany - a cui si aggiungono aree a più giovane vocazione, come California e Napa Valley, che hanno recentemente avviato con successo processi di posizionamento competitivo in ambito enoturistico. Seguono una serie di altri territori a vocazione vinicola che presentano una potenziale evoluzione verso un riconoscimento come destinazione enoturistica Burgundy, Apulia, Chile, Rhone Valley, Australia, La Rioja, Spain - (cfr. Figura 3).

Fig. 3: Le principali destinazioni enoturistiche mondiali

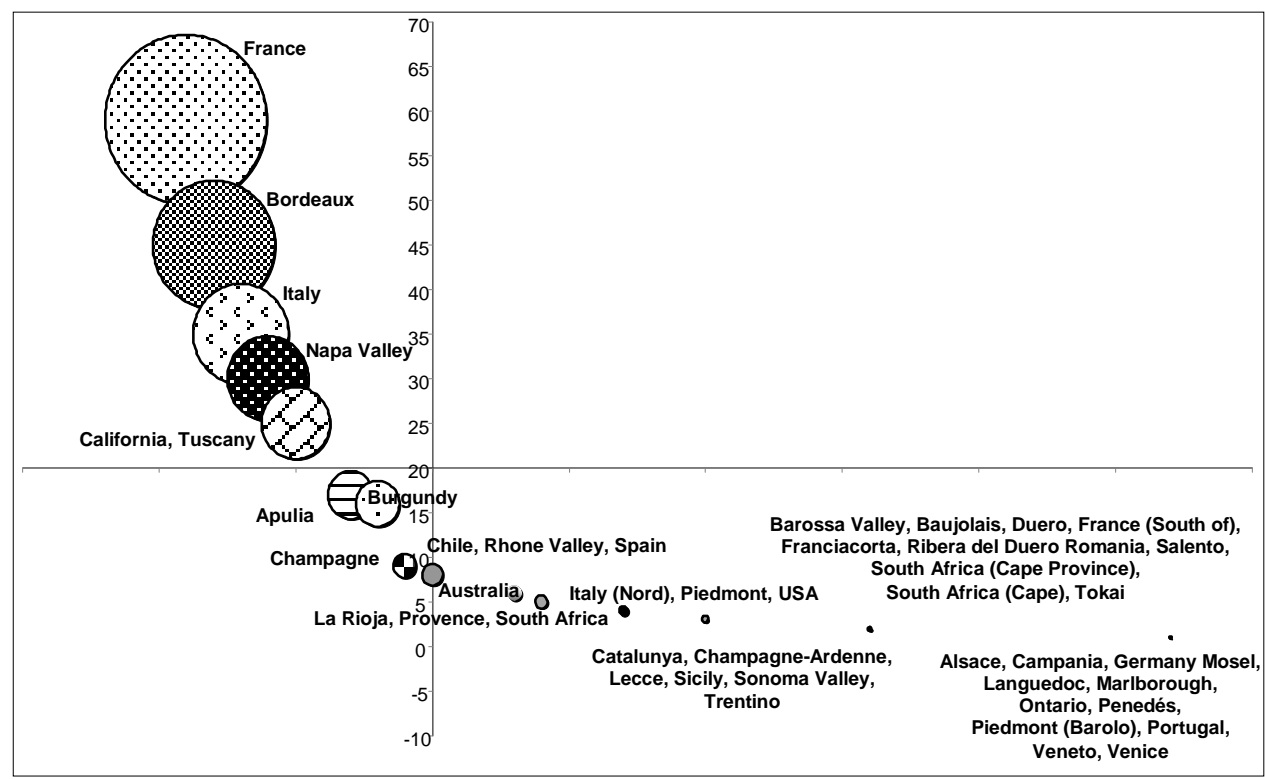

Fonte: ns. elaborazione 
Con riferimento alla notorietà è possibile trarre alcune interessanti osservazioni:

- la dicotomia tra Vecchio e Nuovo Mondo del Vino è ancora molto forte nell'immaginario del consumatore;

- il concetto di destinazione è associato indistintamente alla nazione o ad un territorio geograficamente meno ampio: France - Bordeaux; Italy -Tuscany; California - Napa Valley;

- $\quad$ i wine expert ed i wine enthusiast riconoscono maggiormente le destinazioni enoturistiche geograficamente circoscritte (Napa Valley 77\%/California $44 \%$, Tuscany $60 \%$ /Italy $44 \%$ ).

b) Per la definizione degli attributi dell'immagine percepiti dagli enoturisti, sono state selezionate le prime 6 destinazioni - France, Bordeaux, Italy, Tuscany, California e Napa Valley - impiegando la medesima metodologia utilizzata per pervenire alla mappatura delle risorse riferibili ad una generica destinazione enoturistica (cfr. paragrafo 4.2). Sono stati, dunque, individuati i principali driver dell'immagine di ciascuna di esse attraverso un processo di contestualizzazione delle parole rispetto alla destinazione stessa (per cui Champagne per la France assume l'accezione generica assegnata a wine nel contesto internazionale). Successivamente, ciascun driver, tenendo conto delle direttrici del modello adottato, è stato assegnato al macrogruppo di appartenenza. La matrice che segue (cfr. figura 4) presenta in modo sintetico l'incidenza che i macrogruppi di driver hanno nelle singole destinazioni poste a confronto.

Il valore complessivo del I quadrante - Functional Characteristics/Attributes- di ciascuna destinazione oggetto di osservazione evidenzia la presenza di caratteri comuni che ruotano attorno al concetto di wine ed alle caratteristiche geonaturalistiche e climatiche del territorio. Nell'avvio del processo di emersione, pertanto, la rilevanza di tali fattori presenta differenziali molto bassi tra un territorio e l'altro. Più interessante è, invece, osservarne la composizione. Confrontando, ad esempio, gli elementi che compongono i due macro-gruppi per la California e la Napa Valley la rilevanza risulta invertita. La California è riconosciuta principalmente per elementi strettamente connessi al territorio (nature, sun, summer), mentre per la Napa Valley vi è una identità maggiore degli elementi che ruotano attorno all'elemento wine (Cabernet Sauvignon, Opus wine, bold/red wine, vineyard). Ciò, probabilmente, è frutto delle politiche di marketing attivate in tale area la cui chiave di lettura è "Napa Valley/Wine". Per le aree a più antica tradizione, invece, la percezione dei fattori legati alla cultura enoica è più immediata e presentano la medesima rilevanza sia nel concetto di destinazione/nazione (France $23,6 \%$, Italy $15 \%$, ) che di destinazione/territorio geograficamente circoscritto (Bordeaux 22,2\%, Tuscany 13,8\%).

Dall'osservazione del III quadrante - Attributes/Psychological Characteristics- è riscontrabile, con analoga percezione cognitiva in tutte le destinazioni, il fattore comune excellent wine. Per Italy, California, Tuscany e Napa Valley si osserva, inoltre, l'esistenza di una percezione di tipo cognitivo anche della popolazione (nice/lovely); infine elementi quali il legame cibo-vino (good/great food) e vinoterritorio (beautiful vineyard) sono rinvenibili in Tuscany e Napa Valley. 
Fig. 4: Destination image delle principali destinazioni enoturistiche

\begin{tabular}{|c|c|c|c|c|c|c|}
\hline \multicolumn{6}{|c|}{ Functional Characteristics } & \multirow{2}{*}{$\begin{array}{c}\text { /l quadrante } \\
\begin{array}{c}\text { Productive } \\
\text { and }\end{array} \\
\begin{array}{c}\text { Organitional } \\
\text { heritage }\end{array}\end{array}$} \\
\hline & $\begin{array}{l}\text { Core } \\
\text { resources }\end{array}$ & $\begin{array}{l}\text { Phisiography } \\
\text { and Climate }\end{array}$ & & $\begin{array}{l}\text { Landscape } \\
\text { heritage }\end{array}$ & Culture and History & \\
\hline $\begin{array}{c}\text { France } \\
(29,17 \%)\end{array}$ & $\begin{array}{l}23,60 \%- \\
\text { Food, } \\
\text { Champagne, } \\
\text { Burgundy, } \\
\text { Bordeaux }\end{array}$ & $\begin{array}{l}5,60 \% \text { - } \\
\text { Countryside, } \\
\text { Terroir-cru }\end{array}$ & $\begin{array}{c}\text { France } \\
(31,94 \%)\end{array}$ & $\begin{array}{l}8,33 \% \text { - } \\
\text { Village, } \\
\text { Landscape, } \\
\text { Cotè d'Or }\end{array}$ & - & $\begin{array}{c}23,30 \% \text { - Rich } \\
\text { wine heritage, } \\
\text { Professionality, } \\
\text { Product } \\
\text { enhancement, } \\
\text { Family } \\
\text { oriented }\end{array}$ \\
\hline $\begin{array}{l}\text { Bordeaux } \\
(34,44 \%)\end{array}$ & $\begin{array}{l}22,20 \%- \\
\text { Grape, Red } \\
\text { wine, Claret, } \\
\text { Vineyard }\end{array}$ & $\begin{array}{l}12,20 \%- \\
\text { Countryside, } \\
\text { Sunshine }\end{array}$ & $\begin{array}{l}\text { Bordeaux } \\
(30,00 \%)\end{array}$ & $\begin{array}{l}3,30 \%- \\
\text { Antique } \\
\text { vineyard }\end{array}$ & $\begin{array}{l}21,11 \% \text { - Chateaux, } \\
\text { Famous/interesting } \\
\text { history, Traditional/cultured }\end{array}$ & $\begin{array}{l}5,60 \%- \\
\text { Traditional } \\
\text { winemaking, } \\
\text { Many guided } \\
\text { visited }\end{array}$ \\
\hline $\begin{array}{c}\text { Italy } \\
(24,53 \%)\end{array}$ & $\begin{array}{l}15 \%-\text { Food } \\
\text { Chianti, } \\
\text { Vineyard }\end{array}$ & $\begin{array}{l}9,40 \% \text { - Sun } \\
\text { weather }\end{array}$ & $\begin{array}{c}\text { Italy } \\
(16,98 \%)\end{array}$ & $\begin{array}{l}7,50 \%- \\
\text { Excellent } \\
\text { landscape, } \\
\text { Rolling hill }\end{array}$ & - & $\begin{array}{l}9,40 \% \text { - } \\
\text { Professionality, } \\
\text { Rich wine } \\
\text { heritage }\end{array}$ \\
\hline $\begin{array}{l}\text { Tuscany } \\
(22,50 \%)\end{array}$ & $\begin{array}{l}13,80 \%- \\
\text { Sangiovese, } \\
\text { Chianti, } \\
\text { Vineyard }\end{array}$ & $\begin{array}{l}8,80 \%- \\
\text { Countryside, } \\
\text { Sun }\end{array}$ & $\begin{array}{l}\text { Tuscany } \\
(25,00 \%)\end{array}$ & $\begin{array}{l}12,50 \%- \\
\text { Lush } \\
\text { countryside, } \\
\text { Beautiful } \\
\text { landscape, } \\
\text { Hill town }\end{array}$ & $\begin{array}{l}11,25 \% \text { - History/old, } \\
\text { Ancient properties, Family } \\
\text { generation }\end{array}$ & $\begin{array}{l}3,80 \% \text { - Local } \\
\text { festival, } \\
\text { Excellent } \\
\text { marketing }\end{array}$ \\
\hline $\begin{array}{l}\text { California } \\
(27,08 \%)\end{array}$ & $\begin{array}{l}6,30 \%- \\
\text { Grape, Wine }\end{array}$ & $\begin{array}{l}20,80 \%- \\
\text { Nature, Sun } \\
\text { Summer }\end{array}$ & $\begin{array}{l}\text { California } \\
(27,08 \%)\end{array}$ & 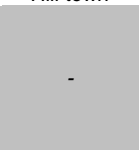 & - & $\begin{array}{c}25,00 \% \text { - } \\
\text { Organization, } \\
\text { Marketing, } \\
\text { New world, } \\
\text { Competitive } \\
\text { new player }\end{array}$ \\
\hline $\begin{array}{l}\text { Napa Valley } \\
(18,29 \%)\end{array}$ & $\begin{array}{c}14,60 \%- \\
\text { Cabernet } \\
\text { Sauvignon, } \\
\text { Opus wine, } \\
\text { Bold red, } \\
\text { Wine, } \\
\text { Vineyard }\end{array}$ & $\begin{array}{l}3,70 \%- \\
\text { Sunshine }\end{array}$ & $\begin{array}{c}\text { Napa } \\
\text { Valley } \\
(25,61 \%)\end{array}$ & $\begin{array}{l}\text { 12,20\% - } \\
\text { Rustic } \\
\text { beauty, } \\
\text { Nice } \\
\text { landscape, } \\
\text { Winery }\end{array}$ & - & $\begin{array}{c}13,40 \%- \\
\text { Organized, } \\
\text { Corporate, } \\
\text { Modern } \\
\text { tourism, Wine } \\
\text { train }\end{array}$ \\
\hline \multicolumn{3}{|c|}{ Emotional attributes } & & \multicolumn{3}{|c|}{ Philosophy and Values } \\
\hline $\begin{array}{l}\text { France } \\
(2,78 \%)\end{array}$ & \multicolumn{2}{|c|}{ Excellent/good wine } & $\begin{array}{c}\text { France } \\
(36,11 \%)\end{array}$ & \multicolumn{3}{|c|}{$\begin{array}{l}\text { Quality, Relaxing, Serenity, Charming, Style, Atmosphere, } \\
\text { Promising, Sophistication, Unforgettable }\end{array}$} \\
\hline $\begin{array}{l}\text { Bordeaux } \\
(2,22 \%)\end{array}$ & \multicolumn{2}{|c|}{ Good/great wine } & $\begin{array}{l}\text { Bordeaux } \\
(33,33 \%)\end{array}$ & \multirow{2}{*}{\multicolumn{3}{|c|}{$\begin{array}{c}\text { Quality, Relaxing Emotion Charming Rhone Style, Open } \\
\text { minded, Romantic, Chic, Exclusive, Serious } \\
\text { Quality, Friendly, relaxing, Sophistication, Fashioned, } \\
\text { Classic, Enthusiastic, Availability, Value for money, } \\
\text { Affordable, Popular, Joy }\end{array}$}} \\
\hline $\begin{array}{c}\text { Italy } \\
(16,98 \%)\end{array}$ & \multicolumn{2}{|c|}{ Excellent/good wine, People } & $\begin{array}{c}\text { Italy } \\
(41,51 \%)\end{array}$ & & & \\
\hline $\begin{array}{l}\text { Tuscany } \\
(12,50 \%)\end{array}$ & $\begin{array}{r}\text { Excellen } \\
\text { Good/great } f \\
p e\end{array}$ & $\begin{array}{l}\text { good wine, } \\
\text { od, Nice/lovely } \\
\text { ople }\end{array}$ & $\begin{array}{l}\text { Tuscany } \\
(37,50 \%)\end{array}$ & \multicolumn{3}{|c|}{$\begin{array}{l}\text { Quality, Friendly, Relaxing, Serenity, Laid back, } \\
\text { Sophistication, Fashioned, Classic, Enthusiastic }\end{array}$} \\
\hline $\begin{array}{c}\text { California } \\
(8,33 \%)\end{array}$ & \multicolumn{2}{|c|}{$\begin{array}{l}\text { Excellent/good wine, } \\
\text { Nice/lovely people }\end{array}$} & $\begin{array}{l}\text { California } \\
(39,60 \%)\end{array}$ & \multicolumn{3}{|c|}{$\begin{array}{c}\text { Promising, Exotic, Welcoming, Fun, Young, Luminous, } \\
\text { Crisp, Busy }\end{array}$} \\
\hline $\begin{array}{l}\text { Napa Valley } \\
(15,85 \%)\end{array}$ & \multicolumn{2}{|c|}{$\begin{array}{l}\text { Excellent/good wine, } \\
\text { Good/great food, Family } \\
\text { friendly, Lovely/beautiful } \\
\text { vineyard }\end{array}$} & $\begin{array}{c}\text { Napa } \\
\text { Valley } \\
(40,00 \%)\end{array}$ & \multicolumn{3}{|c|}{$\begin{array}{l}\text { Friendly, Relaxing, Laid back, Open minded, Chic, Active, } \\
\text { Crowded, Approachable, Expensive, New varied }\end{array}$} \\
\hline
\end{tabular}

Psychological Characteristics

Fonte: ns. elaborazione 
Il confronto dei dati rilevati nel II quadrante Holistic/Functional Characteristics, in cui la valutazione degli attributi funzionali avviene all'interno di un sistema più ampio caratterizzato dalla percezione di legami olistici tra gli stessi, permette alcune interessanti osservazioni:

- il valore complessivo del quadrante delle singole destinazioni, indicativo della rilevanza generale assegnata alle risorse in chiave olistica, è pressoché simile per tutte le aree. Questo evidenzia che nell'immaginario del consumatore gli elementi funzionali hanno legami sistemici che intensificano la loro incidenza, attraverso una differente combinazione dei tre macro-gruppi, nella formazione della brand image;

- il patrimonio paesaggistico - Landscape heritage - è analogamente richiamato sia nelle Old Wine Region che nelle New Wine Region, fatta eccezione per la California $(0 \%)$ per la quale il richiamo avviene nella dimensione Common vs Uniqueness (vineyard, famous area, cost road, San Francisco, Los Angeles);

- il macro-gruppo Culture and History presenta delle peculiarità: per Italy e France il concetto di cultura e storia generico emerge all'interno della direttrice Common vs Uniqueness (Italy/Culture; France/History), mentre il richiamo al legame tra cultura enoica-storia-tradizioni è all'interno del concetto di patrimonio produttivo/organizzativo (France 23,3\%, Italy 9,4\%). I valori si invertono per Bordeaux $(21,11 \%)$ e Tuscany $(11,25 \%)$ per i quali il legame tra cultura-storia-territorio sembra essere più facilmente individuabile. Vengono, infatti, richiamati elementi come chateaux, famous/interesting history, traditional/cultured per Bordeaux così come per la Tuscany history/old, ancient properties, family generation;

- le differenze sono particolarmente rilevanti per quanto concerne la dimensione Productive and organitional heritage, non tanto in termini di rilevanza assegnata agli attributi appartenenti a tale gruppo, quanto per la tipologia che entra a farne parte. È evidente il contrasto tra l'orientamento al marketing, all'organizzazione moderna (wine train) ed alla competitività dei player del Nuovo Mondo (le parole maggiormente richiamate sono: organization, marketing, new world, competitive new player, organized, corporate, modern tourism, wine train) con la tradizione produttiva, il ricco patrimonio enologico, le antiche vigne e la professionalità degli old producers (individuate con le parole: rich wine heritage, professionality, product enhancement, family oriented, traditional winemaking, many guided visited, local festival, excellent marketing).

Il IV quadrante - Holistic/Psychological Characteristics -, che comprende il gruppo Philosophy and Values, è quello più interessante. Il valore complessivo di ciascuna destinazione è risultato per tutte molto al di sopra del valore medio (cfr. figura 2), e ciò dimostra come siano estremamente importanti gli attributi sensoriali e di mood nel processo di emersione della brand image di un territorio. Di rilievo appaiono gli aggettivi che lo caratterizzano: se da un lato in tutte vengono richiamate espressioni descrittive di un'atmosfera tranquilla, amichevole, serena, rilassata, di ritorno alle origini, dall'altro è evidente come ai concetti di fascination, charme e sophisticated delle Old Wine Region si sostituiscono aggettivi come open 
minded, welcoming, fun, young che esprimono il carattere più gioviale e moderno delle New Wine Region.

Un'ultima osservazione concerne la dimensione Common Vs Uniqueness. Per le regioni di più antica tradizione vinicola (France, Italy, Bordeaux e Tuscany) il fil rouge dell'immagine rimane la storia, la tradizione ed il loro legame con il vino; i dati hanno permesso di rilevare che:

- wine è identificato direttamente con denominazioni specifiche come: Champagne, Bordeaux, Burgundy, Bourgogne, Chianti;

- il legame tra wine e food è ben percepito: cheese and wine, fine restaurants, cuisines sono elementi spesso richiamati;

- nella dimensione Uniqueness un ruolo primario gioca il patrimonio storico architettonico (Tour Eiffel per la France, città d'arte e musei per Tuscany).

Per le aree più giovani (California e Napa Valley), invece, assumono maggiore importanza i riferimenti alle grandi metropoli americane (San Francisco, Los Angeles), a specifici siti ambientali (Sonoma Valley Coast, Red Wood Forest) e, soprattutto per la Napa Valley, a servizi quali: wine train, shopping, easy travelling, golf; elementi, questi, indicativi di una visione di marketing orientata a far fruire con facilità il territorio anche offrendo servizi collaterali.

\section{Principali risultati e implicazioni manageriali}

L'elemento di originalità del presente lavoro attiene alla scelta di applicare una metodologia di analisi consolidata in tema di immagine della destinazione ad un ambito, quello enoturistico, di crescente rilevanza in chiave di sviluppo turistico, ma ancora poco studiato ed approfondito. Nonostante i risultati non possano essere generalizzati, per il limite rappresentato dalla difficoltà di definire il campione di indagine secondo idonee regole statistiche, essi hanno comunque fornito un quadro d'insieme di attributi, benefici e atteggiamenti di un'importante categoria di enoturisti qual è quella degli utenti del Web 2.0. In particolare dall'analisi è scaturito un modello concettuale di brand image che evidenzia come gli enoturisti/user del Web 2.0 percepiscano l'immagine di una destinazione enoturistica attraverso un processo organico attivato dal riconoscimento di risorse comuni a tutte le wine region (Core resources, Phisiography and climate). Esse, attraverso un processo di astrazione psicologica, divengono Emotional attributes e perdono, quando percepiti in chiave olistica, la dimensione di semplici attributi per divenire veri attrattori (Landscape heritage, Culture and history, Productive and organizational heritage). Infine, la valutazione olistico-psicologica attiva elementi simbolici ed emozionali (Philosophy and values) generando un sistema integrato all'interno del quale ciascuna componente, relazionandosi con le altre, si arricchisce di immaterialità, traendone valore in termini di riconoscibilità all'esterno. Proprio quest'ultimo passaggio fa sì che l'immagine di una destinazione non sia la semplice somma di attributi, ma la risultante di un ingranaggio di relazioni attivato o percepito dal consumatore. 
L'importanza di un'analisi circolare e sinergica delle dimensioni (Attributes Vs Holistic, Functional Vs Psychological characters, Common Vs Uniqueness) del modello a matrice proposto viene corroborata dalle osservazioni relative alle destinazioni enoturistiche in via di compimento. Si rileva a tal proposito che:

1. tradition, road trip, antique vineyards rappresentano elementi di riconoscibilità della destinazione per aree come il Burgundy;

2. le caratteristiche funzionali (sangria, language, Barcellona) e psicologiche (passion, delight, simpathy, young), unitamente a quelle uniche e distintive che caratterizzano la Spagna (Barcellona, Plaza de Toros, Andalusia) ne fanno più una destinazione turistica che enoturistica; viene, invece, percepita come tale l'area de La Rioja della quale si evidenzia il legame con la tradizione (ancient wineries, tradition, historical and expert);

3. la percezione dell'immagine del South Africa passa attraverso elementi come vineyard, sun, lovely vineyards, local festival, friendly che sembrerebbero conferirgli un riconoscimento in ambito enoturistico. Si evidenzia comunque che non esistono richiami in tale senso tra le risorse distintive, l'unicità della destinazione è infatti ancorata ad un elemento stereotipato come il safari;

4. il Cile sembra essere una destinazione enoturistica emergente, conosciuta grazie al passaparola di amici ed il cui elemento di unicità è lo scenario;

5. per l'Australia gli elementi che caratterizzano l'immagine sono legati tanto al vino (Oak Chardonnay), quanto all'atmosfera (fun); il principale elemento di unicità indicato è la Sydney Opera House.

Dalle osservazioni può rilevarsi come la brand image possa divenire fattore critico di successo capace di agire in termini di promozione e sviluppo di un territorio e delle sue produzioni se, in un'ottica di destination management, le politiche di brand identity tendono a valorizzare e a potenziare l'ingranaggio all'interno del quale si forma la brand image di una wine tourism destination al fine di generare unicità, riconoscibilità e valore.

Il quadro di riferimento descritto può considerarsi, pertanto, esemplificativo degli elementi che dovranno essere consolidati ai fini di un rafforzamento della competitività delle destinazioni enoturistiche, nonché uno strumento per facilitare la discussione e la comunicazione tra gli attori di un territorio in termini di:

- definizione delle strategie tese a rafforzare la dotazione delle componenti immateriali sulla base della loro capacità di formazione dell'immagine;

- indirizzo dei territori verso la co-evoluzione del loro valore e di quello delle proprie produzioni.

\section{Bibliografia}

AAKER D.A. (1996), "Measuring brand equity across products and markets", California Management Review, vol. 38, n. 3, pp. 102-120.

AHMED Z.(1991), "The Influence of the Components of a State's Tourist Image on Product Positioning Strategy", Tourism Management, vol. 12, n. 4, pp. 331-340. 
BAKER B. (2007), Destination branding for small cities: The essentials for successful place branding, Creative Leap Books, Portland.

BALOGLU S., MCCLEARY K.W. (1999), "A model of destination image formation", Annals of Tourism Research, vol. 26, n. 4, pp. 868-897.

BARICH H., KOTLER P. (1991), "A Framework for Marketing Image Management”, Sloan Management Review, vol. 32, n. 2, pp. 94-104.

BEERLI A., MARTÍN J.D. (2004), "Factors influencing destination image", Annals of Tourism Research, vol. 31, n. 3, pp. 657-681.

BIGNE E., SANCHEZ M.I., SANCHEZ J. (2001), "Tourism Image, Evaluation Variables and after purchase behaviour: inter-relationship", Tourism Management, vol. 22, n. 6, pp. 607-616.

BLAIN C., LEVY S.E., RITCHIE J.R.B. (2005), "Destination branding: insights and practices from destination management organizations", Journal of Travel Research, vol. 43, n. 4, pp. 328-338.

BUHALIS D. (2000), "Marketing the competitive destination of the future", Tourism Management, vol. 21, n. 1, pp. 97-116.

CAI L.A. (2002), "Cooperative branding for rural destinations", Annals of Tourism Research, vol. 29 , n. 3, pp. 720-742.

CAI L.A., FENG R., BREITER D. (2004), "Tourist purchase decision involvement and information preferences", Journal of Vacation Marketing, vol. 10, n. 2, pp. 138-148.

CHARTERS S., ALI-KNIGHT J. (2002), "Who is the wine tourist?", Tourism Management, vol. 23, n. 3, pp. 311-319.

CHON K. (1990), "The Role of Destination Image in Tourism: A Review and Discussion", The Tourist Review, vol. 45, n. 2, pp. 2-9.

CLIFTON R. (2003), "The future of brands", in Clifton R. (Ed.), Brands and branding, Bloomberg Press, NY, pp. 227-241.

CROMPTON J.L. (1979), "An assessment of the image of Mexico as a vacation destination and the influence of geographical location upon that image", Journal of Travel Research, vol. 17, n. 4, pp. 18-23.

DADGOSTAR B., ISOTALO R.M. (1995), "Content of City Destination Image for NearHome Tourists", Journal of Hospitality and Leisure Marketing, vol. 3, n. 2, pp. 25-34.

DANA C.J., MCCLEARLY K.W. (1995), "Influencing associations' site-selection process", Cornell Hotel and Restaurant Administration Quarterly, vol. 36, n. 2, pp. 61-68.

DANN G.M.S. (1996), "Tourists' images of a destination-an alternative analysis", Journal of Travel and Tourism Marketing, vol. 5, n. 1-2, pp. 41-55.

DOBNI D., ZINKHAN G.M. (1990), "In search of brand image: a foundation analysis", in Goldber M.E., Gorn G., Pollay R.W. (Eds.), Advances in consumer research, Provo, UT: Association for Consumer Research, vol. 17, pp. 110-119.

ECHTNER C.M., RITCHIE J.R.B. (1991), "The meaning and measurement of destination image", Journal of Tourism Studies, vol. 2, n. 2, pp. 2-12.

ECHTNER C.M., RITCHIE J.R.B. (1993), "The measurement of destination image: an empirical assessment", Journal of Travel Research, vol. 31, n. 4, pp. 3-13.

ELLIOT S., PAPADOPOULOS N., KIM S.S., (2011)"An Integrative Model of Place Image: Exploring Relationships between Destination, Product, and Country Images", Journal of Travel Research, vol. 50, n. 5, pp. 520-534.

EMBACHER J., BUTTLE F. (1989), "A repertory grid analysis of Austria's image as a summer vacation destination", Journal of Travel Research, vol. 27, n. 27 (winter), pp. 3-7. 
FLOREK M. (2005), “The country brand as a new challenge for Poland”, Place Branding, vol. 1, n. 2, pp. 205-214.

FOSTER N., JONES E. (2000), "Image versus Identity: Representing and comparing Destination Images Across a Tourist System - The case of Wales", in Robinson M., Evans N., Long P., Sharpley R., Swarbrooke J. (eds.), Management, Marketing and the Political Economy of Travel and Tourism, Center for Travel and Tourism and Business Education Publishers Ltd.

GALLARZA M., GIL I.S., CALDERON H.G. (2002), "Destination Image: Towards a Conceptual Framework", Annals Tourism Research, vol. 29, n. 1, pp. 56-78.

GARTNER W. (1993), "Image Formation Process", Journal of Travel and Tourism Marketing, vol. 2, n. 2/3, pp. 191-215.

GARTNER C.G., HUNT J.D. (1987), “An analysis of state image change over a twelve-year period (1971-1983)", Journal of Travel Research, vol. 26, n. 2, pp. 15-19.

GOODRICH J. (1978), "The Relationship between Preferences For and Perceptions of Vacation Destinations: Application of a Choice Model", Journal of Travel Research, vol. 17 , n. 2, pp. 8-13.

GOVERS R., GO F.M., KUMAR K. (2007), "Promoting tourism destination image", Journal of Travel Research, vol. 46, n. 1, pp. 15-23.

GUNN C.A. (1972), Vacationscape: Designing tourist regions, Austin, Bureau of Business Research, University of Texas.

HALL M., SHARPLES L., CAMBOURNE B., MACIONIS N. (Eds.) (2000), Wine tourism around the world: Development, management and markets, Butterworth-Heinemann, Oxford.

HALL C.M., MITCHELL R.D. (2002). "The tourist terroir of New Zealand wine: The importance of region in the wine tourism experience", in Montanari A., Food and environment: Geographies of taste, Società Geografica Italiana, Roma.

HAUGLAND S.A., NESS H., GRONSETH B.O., AARSTAD J. (2011), "Development of tourism destinations. An integrated multilevel perspective", Annals of Tourism Research, vol. 38, n. 1, pp. 268-290.

HOSANY S., YUKSEL E., UYSAL M. (2006), "Destination image and destination personality: An application of branding theories to tourism places", Journal of Business Research, vol. 59, n. 5, pp. 638-642.

HUNT J.D. (1975), "Image as a Factor in Tourism Development", Journal of Travel Research, vol. 13, n. 3, pp. 1-7.

JAGO L., CHALIP L., BROWN G., MULEST T., ALI S. (2003), "Building events into destination branding: insights from experts", Event Management, vol. 8, n. 1, pp. 3-14.

KAPFERER J.N. (1997), Strategic brand management: Creating and sustaining brand equity long term, (2nd ed.), Kogan Page Limited, London.

KAPLANIDOU K., VOGT C. (2003), Destination branding: concept and measurement, http:www.tourismcenter.msu.edu, Accessed 15.06.11.

KELLER K.L. (1993), "Conceptualizing, measuring and managing customer-based brand equity", Journal of Marketing, vol. 57, n. 1, pp. 1-22.

KIM S., YOON Y. (2003), "The Hierarchical Effects of Affective and Cognitive Components on Tourism Destination Image", Journal of Travel and Tourism Marketing, vol. 14, n. 2, pp. 1-22.

KOTLER P., HAIDER D.H., REIN I. (1993), Marketing Places, The Free Press, New York.

LASSAR W., MIITTAL B., SHARMA A. (1995), "Measuring customer-based brand equity", Journal of Consumer Marketing, vol. 12, n. 4, pp. 11-19. 
LEISEN B. (2001), "Image segmentation: the case of a tourism destination", Journal of Services Marketing, vol. 15, n. 1, pp. 49-66.

MACKAY K.J., FESENMAIER D.R. (2000), "An exploration of cross-cultural destination image assessment", Journal of Travel Research, vol. 38, n. 4, pp. 417-423.

MARTINEZ E., DE CHERNATONY L. (2004), "The effect of brand extension strategies upon brand image", Journal of Consumer Marketing, vol. 21, n. 1, pp. 39-50.

MAYO E.J. (1973), "Regional images and regional travel behaviour", The Travel Research Association Fourth Annual Conference, Proceedings, Sun Valley, Idaho.

MIN HAN C. (1989), "Country Image: Halo or Summary Construct?", Journal of Marketing Research, vol. 26, n. 2, pp. 222-229.

MITCHELL R., HALL C.M., MCINTOSH A. (2000), "Wine tourism and consumer behaviour", in Hall C.M., Sharples L., Cambourne B., Macionis N. (Eds.), Wine tourism around the world: Development, management and markets, Elsevier Science, Oxford.

MORGAN N., PRITCHARD A., PIGGOTT R. (2002), "New Zealand, 100\% pure. The creation of a powerful niche destination brand", Brand Management, vol. 9, n. 4/5, pp. 335-354.

MORRISON A., ANDERSON D. (2002), Destination branding. Available from: http://www.macvb.org/intranet/presentation/DestinationBrandingLOzarks6-10-02.ppt. Accessed 18.06.12.

MURPHY J. (1998), "What is branding?", in Hart S., Murphy J. (Eds.), Brands: The new wealth creator, New York University Press, New York, pp. 1-12.

NANDAN S. (2005), "An exploration of the brand identity-brand image link age: a communications perspective", Brand Management, vol. 12, n. 4, pp. 264-278.

OOI C.S. (2004), "Poetics politics of destination branding: Denmark", Scandinavian Journal of Hospitality Tourism, vol. 4, n. 2, pp. 107-128.

PAPPU R., QUESTER P.G., COOKSEY R.W. (2005), "Consumer-based brand equity and country-of-origin relationships", European Journal of Marketing, vol. 40, n. 5-6, pp. 696-717.

PARENTEAU A. (1995), Marketing Practico del Turismo, Sintesis S.A., Madrid.

PEARCE P.L., (1982) "Perceived Changes in Holiday Destinations", Annals of Tourism Research, vol. 9, pp. 145-164.

PENCARELLI T., GREGORI G.L. (a cura di) (2009), Comunicazione e branding delle destinazioni turistiche. Una prospettiva manageriale, Franco Angeli, Milano.

PETERS G.L. (1997). American winescapes: The cultural landscapes of America's wine country, Westview Press, USA.

PIKE S., RYAN C. (2004), "Destination Positioning Analysis through a Comparison of Cognitive, Affective, and Conative Perceptions", Journal of Travel Research, vol. 2, n. 4, pp. 333-342.

PIKKEMAAT B. (2004), "The measurement of destination image: the case of Austria", The Poznan University of Economics Review, vol. 4, n. 1, pp. 87-102.

REYNOLDS W.H. (1985), "The role of consumer in image building", California Management Review, Spring, pp.69-76.

RIES A., TROUT J. (1972), The Positioning Era Cometh, Advertising Age, New York.

RITCHIE J.R.B., RITCHIE R.J.B. (1998), "The branding of tourism destinations: pas achievements and future challenges", in AIEST. (Ed.), Proceedings of the 1998 annual congress of the International Association of Scientific Experts in Tourism, destination marketing: Scopes and limitations, International Association of Scientific Experts in Tourism, Marrakech, pp. 89-116. 
ROSATO P., IAZZI A., (2008), "Il sistema delle risorse immateriali nei processi competitivi delle destinazioni turistiche", in Atti del XXXIII Convegno AIDEA "Le risorse immateriali nell'economia delle aziende", 24-25 settembre, Ancona.

ROSATO P., SCORRANO P. (2010), "Un modello interpretativo per i sistemi turistici di prodotto. Il caso del STP Puglia Food and Wine", Sinergie Rapporti di Ricerca, n. 31, pp. 217-242.

RYAN C. (2003), Recreational Tourism. Demand and Impact, Channel View Publications, New York.

SCORRANO P. (2011), "The 2.0 marketing strategies for wine tourism destinations of excellence", Chinese Business Review, vol. 10, n. 10, pp. 948-960.

SCORRANO P. (2011), "Wine Tourism Development of the Territory: A Comparative Analysis of Destinations of Excellence", in Conference Proceedings of 12th International Conference "Building Capabilities for Sustainable Global Business: Balancing Corporate Success Social Good", July 21, 22, 23, Singapore.

SONMEZ S., SIRAKAYA E. (2002), "A distorted destination image? The case of Turkey", Journal of Travel Research, vol. 41, n. 2, pp. 185-196.

TAPACHAI N., WARYSZAK R. (2000), "An Examination of the Role of Beneficial Image in Tourist Destination Selection", Journal of Travel Research, vol. 39, n. 1, pp. 37-44.

TASCI A.D.A., KOZAK M. (2006), "Destination brands vs destination images: do we know what we mean?", Journal of Vacation Marketing, vol. 12, n. 4, pp. 299-317.

TELFER D.J. (2001). "From a wine tourism village to a regional wine route: An investigation of the competitive advantage of embedded clusters in Niagara, Canada", Tourism Recreation Research, vol. 26, n. 2, pp. 23-33.

UYSAL M., CHEN J.S.,WILLIAMS D.R. (2000), "Increasing state market share through a regional positioning", Tourism Management, vol. 21, n. 1, pp. 89-96.

WALMSLEY D., YOUNG D. (1998), "Evaluative Images and Tourism: The Use of Personal Constructs to Describe the Structure of Destination Images", Journal of Travel Research, vol. 36, n. 3, pp. 65-69.

WARD S., LIGHT L., GOLDSTINE J. (1999), "What high-tech managers need to know about brand", Harvard Business Review, July-August, pp. 85-95.

WOODSIDE A.G., LYSONSKY S., (1989) "A General Model of Traveller Destination Choice", Journal of Travel Research, vol. 27, n. 4, pp. 8-14. 
